\title{
Robotic Surgery for Salivary Gland Disease
}

\author{
Anne C. Kane $\cdot$ Meghan N. Wilson • \\ Rohan R. Walvekar
}

Published online: 27 April 2014

(C) Springer Science+Business Media New York 2014

\begin{abstract}
Robotic surgery has revolutionized surgical capabilities and continues to change surgical approaches and management. Robotic surgery is in its infancy with respect to head and neck surgery. However, there are several validated indications for robotics in ENT. The authors discuss the role of robotic surgery specifically related to salivary gland disease and discuss the indications, complications and anatomic nuances that justify the current role and continued effort to expand indications for robotic surgery in the field of head and neck surgery.
\end{abstract}

Keywords Robotic surgery $\cdot$ Salivary gland disease . salivary stone $\cdot$ da Vinci surgical system

\section{Introduction}

The development of minimally invasive endoscopic procedures revolutionized the field of surgery. Endoscopicassisted procedures have now become standard over open procedures in many fields, including general surgery, gynecological surgery, cardiac surgery and urology [12]. The subsequent development of robotic surgery has allowed for further improvement of the precision and efficacy offered by endoscopic surgery [1]. Robotic surgery has emerged as a less invasive approach to the surgical

A. C. Kane

School of Medicine, Louisiana State University Health Sciences Center, New Orleans, LA, USA

M. N. Wilson · R. R. Walvekar $(\square)$

Department of Otolaryngology Head Neck Surgery, Louisiana

State University Health Sciences Center, 533 Bolivar Street,

Suite 566, New Orleans, LA 70112, USA

e-mail: rwalve@1suhsc.edu field in certain areas of surgical medicine. It offers the benefits of smaller surgical scars or placement of scars in more cosmetic areas, decreased blood loss, and decreased morbidity and mortality [10].

Robotic surgery has mostly advanced with use of the da Vinci robot system. The da Vinci robot is a telemanipulative surgical system that consists of a surgeon's control console and a surgical arm cart. The surgeon's console is equipped with three-dimensional imaging of the surgical field. The surgeon controls the surgical arm inside the control console, and his/her hand motions are translated through the surgical arm, which mechanically maneuvers the operative instruments and video-endoscope within the operative pocket [1]. The high-resolution, magnified, threedimensional view of the operative field provides a direct line of sight and allows for standardization of the procedure for the surgeon and participating assistants and observers.

Within the field of otolaryngology, the use of robotic technology is still evolving. Transoral robotic surgery (TORS) has been validated for use in the resection of oncologic disease of the tonsil and base of the tongue and described for use for tumors of the oropharynx, hypopharynx, larynx and parapharyngeal spaces $[2-4,5 \bullet \bullet, 6,7]$. Robotic-assisted surgery has also been described for selective neck dissections, removal of thyroid and parathyroid glands using distant access incisions, and obstructive sleep apnea surgery $[8,9,11]$.

Robotic procedures have also been described for salivary gland disorders, including the removal of floor of the mouth ranulas, salivary stones, and salivary tumors of the submandibular gland and oropharynx. TORS offers several advantages over traditional transoral surgery in some areas while offering a comparable platform for surgical intervention compared to standard techniques in other areas. For example, the magnification and dexterity provided by the 
robot allow precise dissection and preservation of delicate structures contained within the oral cavity and floor of the mouth providing comparable surgical handling as compared to the standard approach of manual dissection. TORS offers a line-of-sight view of the anterior and posterior floor of the mouth and the ability for the treatment team to collaborate and participate in the surgery, which is an advantage over the current view offered by traditional transoral surgery. In our experience, TORS has offered better surgical access, diminished blood loss, shorter hospital stay, and lower morbidity and postoperative pain when used for floor of mouth surgery [10]. Because of the preliminary success of robotic surgery in the management of salivary gland diseases and oral cavity tumors, the authors believe further applications for the robotic surgical approach to the management of salivary tumors can be developed. In this article, we will discuss the applications for transoral robotic surgery for management of salivary gland disorders.

\section{Ranula Excision}

A ranula is a type of mucocele in the floor of the mouth that arises from the sublingual gland (SLG). Extravasation of mucus from the SLG typically occurs because of salivary duct obstruction or trauma causing acinar damage [14]. Ranulas typically present as unilateral or occasionally bilateral fluctuant swelling of the floor of the mouth, causing discomfort and difficulty in speech for the patient. Plunging ranulas are a more advanced stage of ranula, where the ranula extends beyond the diaphragm of the floor of the mouth musculature, i.e., the mylohyoid muscle, and extends into the neck. There are both medical and surgical options available for ranula treatment. Ranula excision with the associated SLG has the highest success rate in the prevention of ranula recurrence [14-17]. This is most often accomplished through the transoral approach, which can be used for plunging ranulas as well $[15,16]$.

The challenges of ranula excision and of floor of mouth surgery involve the identification and preservation of Wharton's duct, the lingual nerve and its terminal branches, and the excision of the entire SLG via a transoral route [13]. Transoral excision can be difficult dependent on patient anatomy, surgeon's experience, pathology and number of assistants available. Additionally, the floor of the mouth is a diaphragm that separates the oral cavity from the neck and is a dynamic structure that is always changing during surgical manipulation [13]. The authors conceptualized and reported the use of the da Vinci system for anterior floor of the mouth surgery with some specific advantages in mind. TORS offers a direct line-of-sight view to the anterior floor of the mouth and provides an opportunity to convert this dynamic, unpredictable and multiple assistant-dependent surgical experience to a more predictable, standardized approach where the entire team can participate in the surgical dissection with enhanced visualization and surgical access $[13,18]$.

Once exposure of the field has been achieved, the robotic arms of the da Vinci system are placed into position in the patient's mouth. The surgeon controlling the instruments does so from a control console within the room. The surgeon utilizes a 5-mm Maryland dissecting forceps and a 5-mm monopolar cautery spatula and can interchange dissecting instruments as necessary. The first assistant remains at the head of the bed and provides retraction and suction as necessary. During the surgery, the SLG is dissected and separated from the lingual nerve and Wharton's duct. The lingual nerve is dissected as well along its length to confirm its identity. Once these important structures have been identified and dissected out, the SLG and ranula can be excised, and the floor of the mouth can be re-approximated using absorbable stitches [13].

Complications of excision of the SLG with assistance of TORS are the same as for the standard approach and include injury to the submandibular duct and/or lingual nerve. The senior author of this chapter believes that these complications are more easily avoided with the visualization allowed by the robot in comparison to the standard transoral approach. Our experience with the floor of the mouth robot-assisted surgery has validated this fact and shown that direct line of sight, illumination and magnification allow for a more precise dissection and greater ability to preserve lingual nerve function. If injury of the submandibular duct does occur, the duct can be repositioned and stented for 2-3 weeks. Complete salivary duct function can be restored after stent removal [13].

The use of the TORS for the floor of the mouth surgery provides a more predictable environment, improved exposure, dexterity and less need for multiple assistants while still allowing for preservation of the lingual nerve and Wharton's duct. Further validation is needed to assess long-term recurrence rates and cost effectiveness when compared to traditional procedures [18] (Fig. 1).

\section{Salivary Calculi}

Sialoliths, or salivary stones, are the most common cause of obstructive sialadenitis, followed by stenosis and sialodochitis [19]. Salivary stones are most commonly located in the submandibular gland ( $80 \%$ ), followed by the parotid (19\%) and SLGs (1\%), respectively [20]. Conventional treatment of sialolithiasis is surgical removal of the affected gland. The introduction of sialendoscopy has revolutionized the management of salivary stones and 


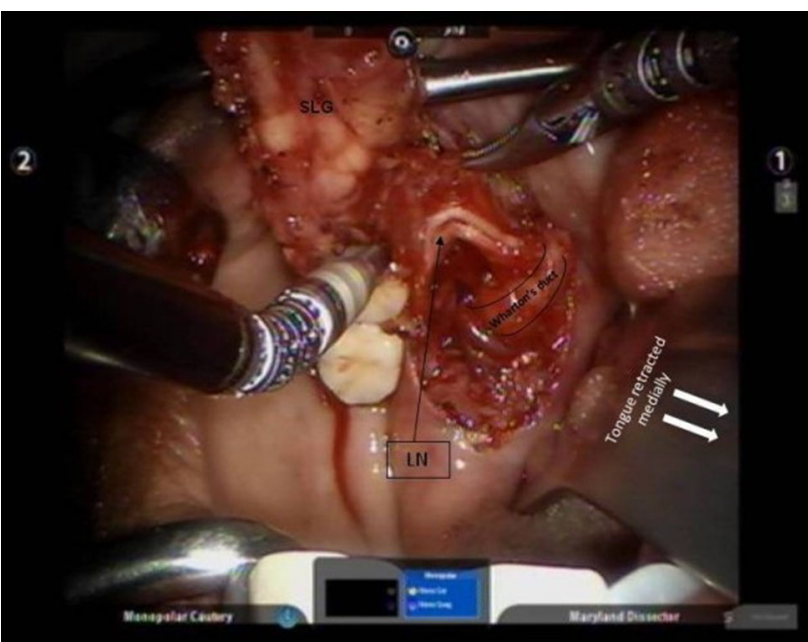

Fig. 1 Dissection of the left sublingual gland $(S L G)$ with identification of the terminal branches of the lingual nerve $(L N)$ and delineation of the Wharton's duct

significantly reduced the rate of major gland removal due to sialolithiasis $[21 \bullet, 22]$.

The majority of salivary stones range between 3 and $7 \mathrm{~mm}$ and can be managed using sialendoscopy, lithotripsy or laser fragmentation $[24,25]$. Larger $(\geq 8 \mathrm{~mm})$ submandibular and parotid stones can be removed using the combined approach technique, which pairs stone localization by sialendoscopy with transoral or external removal of stone(s) [26, 27••, 28]. The combined approach technique has been shown to have a gland preservation rate of $86 \%$ [23]. However, transoral removal of large submandibular stones can be complicated by hilar-glandular location of the stone, limited exposure of the proximal floor of the mouth due to reduced mouth opening, large teeth and obesity. Limited exposure makes removal of stones more difficult and also complicates the identification and preservation of the lingual nerve and the placement of sutures in the salivary duct for repair after sialolithotomy [21•].

Robotic-assisted removal of salivary stones is based on the combined approach technique and utilizes the da Vinci robot system for the open portion of the combined approach procedure. This has been described by Walvekar et al. for the removal of submandibular megaliths, stones

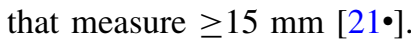

The robotic-assisted removal of a submandibular megalith uses the standard combined approach technique described by Marchal [27••] and utilizes the da Vinci robot system for the dissection of the posterior floor of mouth. Endoscopic localization of the stone is performed using sialendoscopy. Although it is expected that the stone may not be amenable to endoscopic removal, salivary endoscopy allows initial dilation of the duct and stone localization. In addition, salivary endoscopy following stone removal is advantageous for the removal of residual stones and to check the integrity of the salivary duct repair. Dilation and irrigation of the duct and at times intubation of the duct with a stent prior to the open component help in visual localization of the submandibular duct in the mouth floor during robotic dissection.

After the stone has been localized, the open transoral portion of the procedure is begun. A Jennings's mouth gag and tongue retractor are used to expose the oral cavity and surgical site. At this time, the robotic arms of the da Vinci Surgical System are placed into position in the patient's mouth. The first assistant remains at the head of the bed to provide retraction of the tongue and suction, while the primary surgeon operates from within the control console and the second assistant provides external pressure over the submandibular gland. The incision is made over the area of transillumination or pre-marked site. The lingual nerve is dissected along its length and separated from the salivary duct. The Wharton duct containing the stone is then dissected to facilitate identification of the stone. The duct is then mobilized, and the stone within it is isolated with a proximal vessel loop drawing the stone into the field. The duct is incised over the stone, and the stone is delivered. A distal sialendoscopy is then performed to check for additional stones and to remove stone remnants. Wharton's duct is then repaired either via the transoral route or with robotic-assistance over an indwelling salivary stent when indicated. The stent is secured to the mouth floor using a 4-0 nylon single looping stitch described by the senior author $[21 \bullet, 27 \bullet \bullet]$.

This case study confirmed the feasibility of using the da Vinci Si HD Robotic Surgical System for management of giant stones in the submandibular gland with preservation of the lingual nerve and submandibular gland. It concludes that this technique can be used only for larger palpable and impacted stones [21•]. Further experience and data from the author's institution further validates the experience of this case report.

The authors acknowledge the fact that combined approach stone removal is feasible via a transoral route and is often done under local anesthesia in many centers in the world as an in-office procedure. However, when these procedures are done under general anesthesia, operative times and surgical experience can be variable based on patient anatomy, available assistance, stone location and orientation, and surgical access. Our study and experience validate the fact that integration of robotic assistance helps standardize the procedure and surgical exposure, making the procedure more predictable and reproducible across centers in terms of surgical experience, time and outcomes when performed under general anesthesia (Fig. 2).

\section{Submandibular Gland Resection}

Surgical excision of the submandibular gland (SMG) is commonly indicated in patients with neoplasms, chronic 

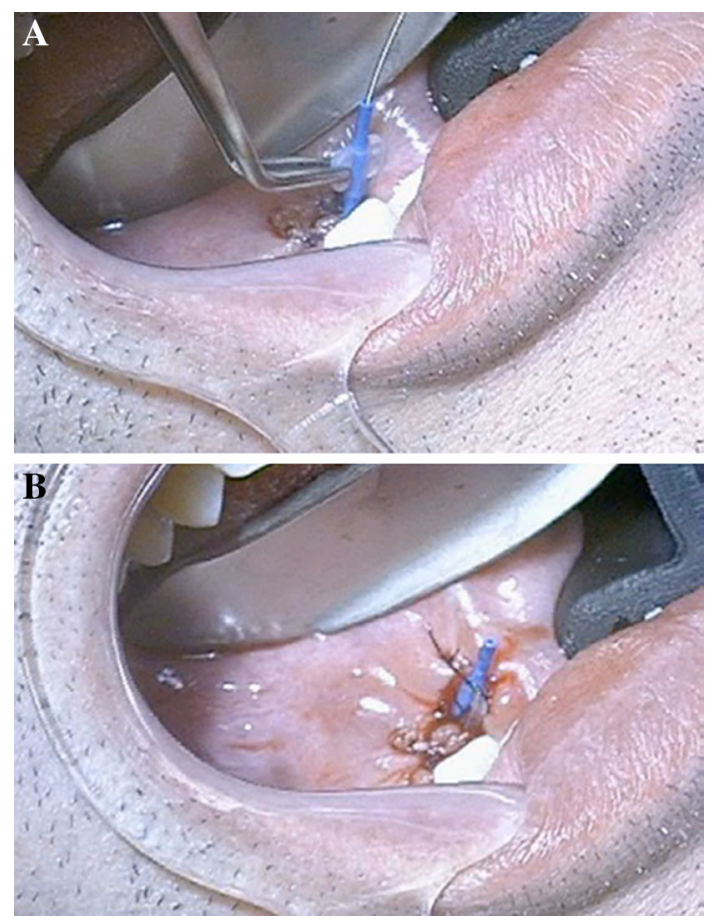

Fig. 2 a Insertion of the salivary stent $1.2 \mathrm{~mm}$ over the guidewire. b Securing the stent to mouth floor with a single loop stitch using 4-0 nylon

sialadenitis and sialolithiasis, and to manage chronic drooling (sialorrhea) unresponsive to conservative treatment [29]. Classic submandibular sialadenectomy utilizes a trans-cervical approach [3]. Risks of conventional open surgery include infection, hematoma, salivary fistula, and temporary or permanent damage to the marginal mandibular, lingual or hypoglossal nerve [30-32]. Other open approaches for SMG removal include the submental, transoral and retroauricular. These alternative approaches offer advantages in terms of cosmesis over the traditional transcervical approach [29].

Minimally invasive approaches, including endoscopic and robotic-assisted approaches, offer improved cosmesis, less scarring, diminished blood loss, shorter hospital stay and lower morbidity compared to traditional open approaches [29]. Endoscopic approaches have been incorporated into the transoral, submental and transcervical approaches and have been determined to be feasible in human patients [29, 30, 33-35, 49]. The retroauricular approach has been described and conducted endoscopically and with robotic assistance in humans [36, 37•, 38], and an endo-robotic approach has also been described in a cadaver model [1].

The application of endoscopic and robotic-assisted surgery in the neck initially faced several challenges because of the lack of a discrete, anatomically distinct neck "cavity" and the relative need for sufficient maneuverable space for endoscopic instrumentation [1]. Additionally, early investigations in animals were complicated by injuries to neurovascular structures, pneumothorax, and increased pressure on the jugular vein and carotid sinus [1, 39, 40]. Terris et al. developed a hybridized technique utilizing balloon dissection to first create an operative pocket and low pressure $\mathrm{CO}_{2}$ to insufflate and maintain the pocket. This technique avoids the complications of high pressure insufflations [41, 42], and better results have been achieved with the creation of this operative pocket, which allows better visualization and access to vital structures of the neck [1] (Fig. 3).

Robotic-assisted SMG resection was first described in porcine and cadaver models. Haus et al. completed three unilateral submandibular resections in the porcine model in 2003 and found that operative times were decreased with the robotic-assisted surgery when compared to a prior study utilizing an endoscopic-assisted approach in the porcine model $[10,41]$. They did not appreciate any gross or histologic injuries to the specimens [10].

Terris et al. [42] described successful endoscopic resection of the SMG in a cadaver model using balloon dissection and low pressure carbon dioxide insufflation. The endo-robotic resection of the SMG described by Terris et al. in the cadaver model in 2005 used three 10- to 14-mm lower neck incisions. The operation was described in three stages. An operative pocket was created in the subplatysmal plane with placement of the central trocar over the clavicular head of the sternocleidomastoid muscle. Trocars were aimed toward the SMG and maintained by compressed air insufflated at a pressure of $4 \mathrm{mmHg}$. Two 7-mm trocars and a 12-mm stereoscopic camera were inserted for use during the procedure. The SMG was approached inferiorly in the subplatysmal plane. Care was taken to avoid damage to the marginal mandibular nerve, while the facial artery and vein were identified, isolated and then ligated using robotic clip appliers. The lingual and hypoglossal nerves were also identified and preserved. The SMG was mobilized and retrieved through the 14-mm trocar incision. This study by Terris et al. found that the technique was feasible and decreased operative times when compared to endoscopic approaches [1]. Possible adverse effects of this approach include subcutaneous emphysema, injury to the marginal mandibular, hypoglossal or lingual nerves, and other neural and vascular structures of the neck in addition to conventional risks [1].

The retroauricular approach provides improved cosmesis by elimination of a neck scar. The scar is easily hidden behind the auricle and underneath hair. Lee et al. used the retroauricular approach for removal of the SMG for five patients with pleomorphic adenoma. The incision is made in the lower portion of the postauricular sulcus arching toward the middle to upper third of the sulcus then arching toward 
Fig. 3 Cervical incisions (postauricular, lateral transcervical and submental) for approaches to the submandibular gland (a frontal view; b lateral view; c submental view)

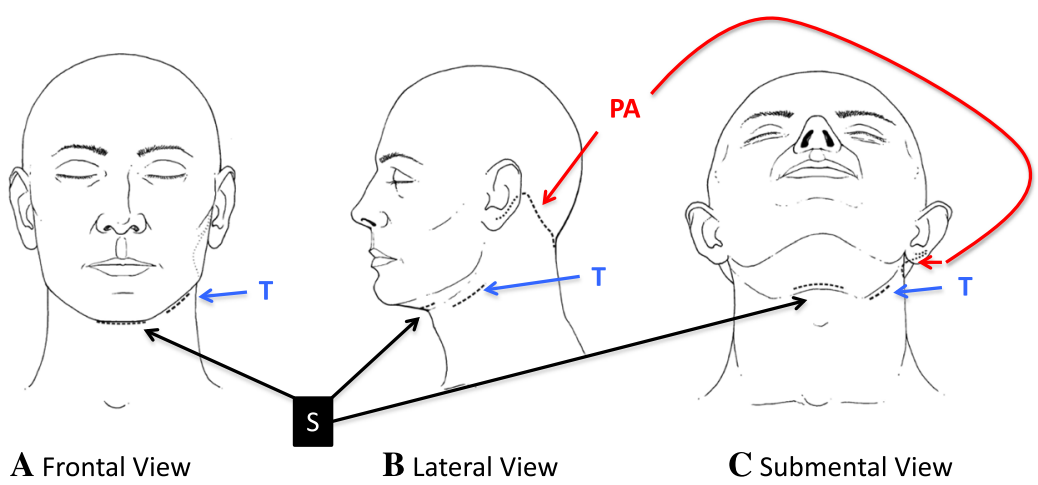

Post Auricular Incision - PA

Lateral Trans-cervical Incision - T Submental Incision - S

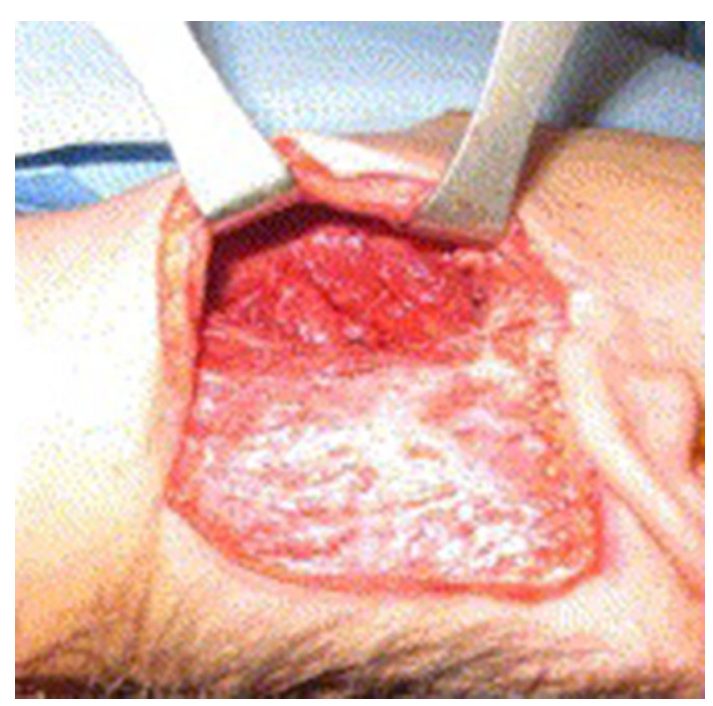

Fig. 4 Retroauricular approach, view showing elevated skin flap

the hairline. A subplatysmal flap is then raised on top of the sternocleidomastoid muscle and continued anteriorly to the midline of the neck and the inferior margin of the mandible. The SMG is exposed, and the skin flap is raised using a lifting device to make a working space. The da Vinci Surgical System with an endoscopic arm and two instrument arms is then introduced. Gland dissection is begun at the inferior portion of the gland. Care was taken to identify and preserve the lingual and hypoglossal nerves. The marginal mandibular nerve was also identified, and Wharton's duct was ligated during the procedure. Preservation of the gland capsule was achieved in areas of tumor penetration in order to prevent tumor spillage [37•] (Fig. 4).

Lee et al. found that the retroauricular approach was useful in exposing the upper neck and providing sufficient working space for robotic instrumentation. The patients were all satisfied with the cosmetic result, despite having a 4-6-cm incision, which is longer than the traditional open transcervical incision. They also found that this technique had decreased operative times compared to endoscopic SMG resection. They did not report any postoperative complications including paralysis or paresthesias of the lingual nerve, hypoglossal nerve or the marginal mandibular branch of the facial nerve. Their mean follow-up time was 5 months, and during that time period, none of their subjects had tumor recurrences. There is still a need for an evaluation of a larger patient group with a longer follow-up period to determine safety and oncologic outcomes [37•].

A similar study done by DeVirgilio et al. [38] performed robotic sialadenectomy of the SMG with a modified facelift approach in five patients with benign pathologies. They used a similar operative approach as Lee et al. except that they initially created a supra-platysmal flap prior to encountering the SMG. This was done to avoid injury to the marginal mandibular nerve. This study did have increased operative times when compared to Lee et al.; however, both studies acknowledged that operative times decreased as the surgeon and assistant become more proficient at the procedure [37•,38].

The transoral approach involves a floor of mouth incision to access the gland. Advantages of this approach include decreased risk of injury to the marginal mandibular nerve, avoidance of an external scar, minimal risk of postoperative sialocele formation and inflammation of Wharton's duct. Disadvantages include scar contracture in the floor of the mouth and injury to the lingual and hypoglossal nerves [33]. The endoscopic transoral approach has an additional advantage of improved visualization, magnification and illumination of the anatomical landmarks. It also creates a wider surgical field compared to the open approach. Disadvantages include the cost of endoscopic equipment, the learning curve of the surgeon and the need for increased operative and setup time [29]. Recent 


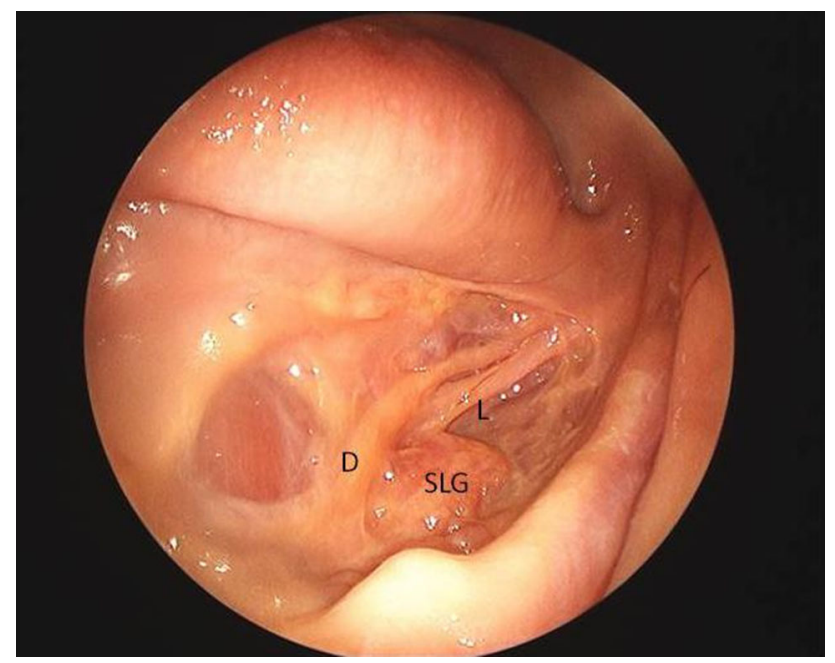

Fig. 5 Transoral approach. Endoscopic view of the transoral approach showing submandibular duct $(D)$, lingual nerve $(L)$ and sublingual gland $(S L G)$

literature has confirmed the transoral endoscopic approach as a feasible means for SMG removal in cases of benign disease $[33,43,49]$. Due to the preliminary success of the endoscopic transoral procedure, there is a potential application of TORS for transoral SMG removal. Use of TORS could potentially overcome the reduced operative field between the tongue and the mandible as well as prevent injury to the lingual or hypoglossal nerves through delicate dissection of these structures (Fig. 5).

\section{Minor Salivary Gland Tumors}

Tumors of the salivary glands account for up to $6 \%$ of head and neck neoplasms [44]. They can occur in the major or minor salivary glands. Major salivary glands include the parotid, submandibular and SLGs. The minor glands are comprised of a group of approximately 600-1,000 small glands, which are dispersed throughout the upper aerodigestive submucosa, which includes the palate, lips, pharynx, nasopharynx, larynx and parapharyngeal space. While the majority of major salivary gland tumors are benign, the opposite is true for minor salivary gland tumors, with the majority being malignant [45]. Minor salivary gland tumors comprise $2-3 \%$ of all salivary tumors [46]. The most common type of minor salivary malignancies includes adenoid cystic, mucoepidermoid and acinic cell carcinomas [47]. Traditional approaches for excision of minor salivary gland neoplasms include the translabial, transmandibular and transcervical. These approaches are associated with significant morbidities, including jaw nonunion, malocclusion, palate-related complications and additional surgical ablative complications, which include dysphagia and altered speech. The successful application of the minimally invasive TORS approach for management of SCC of the oropharynx has allowed the opportunity for management of minor salivary gland tumors by this same approach.

A retrospective review conducted by Villanueva et al. [48] at Mount Sinai followed ten patients over a 5-year period. The study group had either T1 or T2 cancers of the minor salivary glands, located in the base of the tongue, tonsil, soft palate or lateral pharyngeal wall. Short-term follow-up data demonstrated safety and feasibility for the management of minor salivary tumors of the oropharynx as well as low morbidity relative to open procedures. Preliminary oncologic outcomes in this limited group were encouraging. No patients in this group suffered surgical complications, and all patients achieved good functional outcomes. There is a need for additional investigations with larger numbers of subjects and long-term follow-up to determine oncologic outcomes and cost effectiveness.

The disadvantages of robotics include the high cost of the da Vinci robot system and the need for further cost analysis for these procedures. TORS does have the same risks as conventional transoral surgery; however, we believe that these risks are reduced because of better visualization and more precise dissection using the da Vinci instruments. Robotic surgery in the neck includes conventional risks as well as the risk of subcutaneous emphysema, injury to the marginal mandibular, hypoglossal and the lingual nerves, and other neural and vascular structures of the neck [1]. However, the hybridized technique described above aims to, and has so far succeeded in, reducing these risks [41]. The da Vinci robotic system also requires an adequate learning curve for the surgeon and assistant, which can affect operative time for less experienced surgeons [38].

\section{Future Applications/Conclusions}

The application of the TORS for the head and neck as well as salivary gland tumors is evolving and is in many ways is still in its infancy. Technological advances have allowed us the opportunity to consider more functional surgeries for tumors of the oropharynx and allowed us to extend these applications to other areas such as the salivary glands.

In general, numerous studies have shown a decrease in morbidity and mortality with overall preservation of important structures due to the improved visualization and the delicacy and dexterity of the robotic arm manipulation of tools while dissecting vital structures. Several studies have shown decreased operative times, improved cosmesis, and promising functional and oncologic outcomes [1, 10, $21 \bullet, 37 \bullet, 48]$. Robotic-assisted surgery also provides an opportunity for the surgeon and assistants to work in 
tandem, as all surgical steps are visualized by the surgical team and the operating room staff. This provides an advantage for teaching residents, medical students and OR staff in normally poorly visualized surgical fields. There is also the future potential to provide healthcare in underserved areas if the surgeon can perform the surgery from a distant location and be assisted by personnel in the underserved surgical center.

The current applications of robotic surgery for salivary gland disorders need further evaluation with long-term studies to validate its utilization and cost. In addition, such studies will help us determine the benefit that this technology offers to our patients in terms of outcomes and preservation of function.

\section{Compliance with Ethics Guidelines}

Conflict of Interest Anne Kane and Meghan N Wilson declare that they have no conflict of interest. Rohan R Walvekar declares no conflict of interest with respect to robotic surgery and related industry. Rohan R Walvekar is a consultant for Hood Laboratories, Pembroke, USA that manufactures salivary stents.

Human and Animal Rights and Informed Consent This article does not contain any studies with human or animal subjects performed by any of the authors.

\section{References}

Papers of particular interest, published recently, have been highlighted as:

- Of importance

•- Of major importance

1. Terris DJ, Haus BM, Gourin CG, Lilagan PE. Endo-robotic resection of the submandibular gland in a cadaver model. Head Neck. 2005;27(11):946-51.

2. O'Malley BW, et al. Transoral robotic surgery (TORS) for base of tongue neoplasms. The Laryngoscope. 2006;116(8):1465-72.

3. Park YM, et al. Transoral robotic surgery (TORS) in laryngeal and hypopharyngeal cancer. J Laparoendosc Adv Surg Tech A. 2009;19(3):361-8.

4. O'Malley BW, et al. Transoral robotic surgery for parapharyngeal space tumors. Orl J Otorhinolaryngol Relat Spec. 2010;72(6):332-6.

5. - Weinstein GS, et al. Transoral robotic surgery: radical tonsillectomy. Arch Otolaryngol Head Neck Surg. 2007;133(12): 1220-6. This article is a landmark article that describes the utility of robotic instrumentation for oropharyngeal cancers and also touches on relevant anatomy that is important for salivary gland tumor management or floor mouth surgery.

6. Lee HS, et al. Transoral robotic surgery for huge spindle cell lipoma of the hypopharynx. J Craniofac Surg. 2013;24(4):1278-9. doi:10.1097/SCS.0b013e3182860361.

7. Lallemant B, et al. Transoral robotic surgery for the treatment of T1-T2 carcinoma of the larynx: preliminary study. Laryngoscope. 2013;123(10):2485-90. doi:10.1002/lary.23994.
8. Cognetti DM, et al. Early adoption of transoral robotic surgical program: preliminary outcomes. Otolaryngol Head Neck Surg. 2012;147(3):482-8. doi:10.1177/0194599812443353.

9. Landry CS, et al. Robot assisted transaxillary surgery (RATS) for the removal of thyroid and parathyroid glands. Surgery. 2011;149(4):549-55.

10. Haus BM, Kambham N, Le D, et al. Surgical robotic applications in otolaryngology. Laryngoscope. 2003;113(7):1139-44.

11. Lin HS, et al. Transoral robotic surgery for treatment of obstructive sleep apnea-hypopnea syndrome. Laryngoscope. 2013;123(7):1811-6. doi:10.1002/lary.23913.

12. Clayburgh DR, et al. Surgical Innovations. Otolaryngol Clin North Am. 2013;46(4):615-28. doi:10.1016/j.otc.2013.04.003.

13. Walvekar, RR. Gary, CC, Williams, AP. Oliver, PD. "Transoral Robotic Surgery for Ranula Excision" Operative Atlas of Otorhinolaryngology Head and Neck Surgery: Volume 2. Ed. Bachi T Hathiram, Vicky S Khattar. New Delhi: Jaypee Brothers Medical Publishers. 2013: 1649-1653.

14. Harrison JD. Modern management and pathophysiology of ranula: literature review. Head Neck. 2010;32(10):1310-20.

15. Huang SF, et al. Transoral approach for plunging ranula-10yearexperience. Laryngoscope. 2010;120(1):53-7.

16. Patel MR, Deal AM, Shockley WW. Oral and plunging ranulas: what is the most effective treatment? Laryngoscope. 2009;119(8):1501-9.

17. Zhao YF, et al. Clinical review of 580 ranulas. Oral Surg Oral Med Oral Pathol Oral Radiol Endod. 2004;98(3):281-7.

18. Walvekar, et al. Robotic-assisted transoral removal of a bilateral floor of mouth ranulas. World J Surg Oncol. 2011;9:78.

19. Koch M, Zenk J, Bozzato A, et al. Sialoscopy in cases of unclear swelling of the major salivary glands. Otolaryngol Head Neck Surg. 2005;133:863-8.

20. Witt RL, Iro H, Koch M, McGurk M, Nahlieli O, Zenk J. Minimally invasive options for salivary calculi. Laryngoscope. 2012;122(6):1306-11. doi:10.1002/lary.23272.

21. - Walvekar RR, Tyler PD, Tammareddi N, Peters G. Roboticassisted transoral removal of a submandibular megalith. Laryngoscope. 2011;121:534-7. This article is the first published report on the use of robot for the management of hilar-glandular stones. The article describes in details the procedure and discusses advantages and disadvantages provided by robotic instrumentation for floor of mouth surgery.

22. Kopeć T, Szyfter W, Wierzbicka M, Młodkowska A, Kałużny J. Sialendoscopy in treatment of sialolithiasis-our own experience based on group of 95 patients. Otolaryngol Pol. 2012;66(4 Suppl):11-4. doi:10.1016/S0030-6657(12)70778-3.

23. Wallace E, Tauzin M, Hagan J, Schaitkin B, Walvekar RR. Management of giant sialoliths: review of the literature and preliminary experience with interventional sialendoscopy. Laryngoscope. 2010;120:1974-8.

24. Bodner L. Giant salivary gland calculi: diagnostic imaging and surgical management. Oral Surg Oral Med Oral Pathol Oral Radiol Endod. 2002;94:320-3.

25. Raif J, Vardi M, Nahlieli O, Gannot I. An Er:YAG laser endoscopic fiber delivery system for lithotripsy of salivary stones. Lasers Surg Med. 2006;38:580-7.

26. Walvekar RR, Bomeli SR, Carrau RL, Schaitkin B. Combined approach technique for the management of large salivary stones. Laryngoscope. 2009;119:1125-9.

27. • Marchal F. A combined endoscopic and external approach for extraction of large stones with preservation of parotid and submandibular glands. Laryngoscope. 2007;117:373-7. This article by Professor Marchal is a landmark article describing the transoral combined approach technique that serves as the basis gland preserving surgery for hilar and glandular stones. It is also necessary to understand the procedure via this article to be able 
to understand the relevance of the introduction of the robotic instrumentation to augment the combined technique.

28. Kopeć T, Szyfter W, Wierzbicka M. Sialendoscopy and combined approach for the management of salivary gland stones. Eur Arch Otorhinolaryngol. 2013;270(1):219-23. doi:10.1007/ s00405-012-2145-x.

29. Beahm DD, Peleaz L, Nuss DW, et al. Surgical approaches to the submandibular gland: a review of literature. Int J Surg. 2009;7:503-9.

30. Baek $\mathrm{CH}$, Jeong HS. Endoscope-assisted submandibular sialadenectomy: a new minimally invasive approach to the submandibular gland. Am J Otolaryngol. 2006;27(5):306-9.

31. Berini-Aytes L, Gay-Escoda C. Morbidity associated with removal of the submandibular gland. J Craniomaxillofac Surg. 1992;20:216-9.

32. Milton CM, Thomas BM, Bickerton RC. Morbidity study of submandibular gland excision. Ann R Coll Surg Engl. 1986;68:148-50.

33. Guerrissi JO, Taborda G. Endoscopic excision of the submandibular gland by an intraoral approach. J Craniofac Surg. 2001;12:299-303.

34. Chen MK, Su CC, Tsai YL, Chang CC. Minimally invasive endoscopic resection of the submandibular gland: a new approach. Head Neck. 2006;28(11):1014-7.

35. Chen LS, Zhang SY, Huang XM, Sun W, Luo XN, Zhan JD, Lu $\mathrm{ZM}$, Song XH. A comparison between endoscopic-assisted submandibular gland resection via retroauricular hairline incision and conventional submandibular gland resection. Zhonghua Er Bi Yan Hou Tou Jing Wai Ke Za Zhi. 2011;46(7):561-5.

36. Kim HS, Chung SM, Pae SY, Park HS. Endoscope assisted submandibular sialadenectomy: the face-lift approach. Eur Arch Otorhinolaryngol. 2011;268(4):619-22. doi:10.1007/s00405-010-1392-y.

37. - Lee HS, Park DY, Hwang CS, et al. Feasibility of robot-assisted submandibular gland resection via retroauricular approach: preliminary results. Laryngoscope. 2012;. doi:10.1002/lary.23321. This paper discusses the retro auricular approach and use of robotic instrumentation for management of submandibular gland lesions. Its provides an alternative to traditional submandibular gland surgery.

38. De Virgilio A, Park YM, Kim WS, Lee SY, Seol JH, Kim SH. Robotic sialoadenectomy of the submandibular gland via a modified face-lift approach. Int $\mathbf{J}$ Oral Maxillofac Surg. 2012;41(11):1325-9. doi:10.1016/j.ijom.2012.04.008.

39. Carreno OJ, Wilson WR, Nootheti PK. Exploring endoscopic neck surgery in a porcine model. Laryngoscope. 1999;109:236-40.

40. Rubino F, Pamoukian V, Shu JF, Deutsch H, Inabnet WB, Gagner M. Endoscopic endocrine neck surgery with carbon dioxide insufflation: the effect on intracranial pressure in a large animal model. Surgery. 2000;128:1035-42.

41. Monfared A, Saenz Y, Terris DJ. Endoscopic resection of the submandibular gland in a porcine model. Laryngoscope. 2002;112:1089-93.

42. Terris DJ, Haus BM, Gourin CG. Endoscopic neck surgery: resection of the submandibular gland in a cadaver model. Laryngoscope. 2004;114:407-10.

43. Chen LS, Zhang SY, Huang XM, Han ZJ, Luo XN, Song XH, Zhan JD. Preliminary experience with endoscope-assisted transoral excision of the submandibular gland. Zhonghua Er Bi Yan Hou Tou Jing Wai Ke Za Zhi. 2011;46(2):149-51.

44. Stenner M, Klussmann JP. Current update on established and novel biomarkers in salivary gland carcinoma pathology and the molecular pathways involved. Eur Arch Otorhinolaryngol. 2009;266(3):333-41.

45. Iyer NG, Kim L, Nixon IJ, et al. Factors predicting outcome in malignant minor salivary gland tumors of the oropharynx. Arch Otolaryngol Head Neck Surg. 2010;136:1240-7.

46. Jones AS, Beasley NJ, Houghton DJ, Helliwell TR, Husband DJ. Tumours of the minor salivary glands. Clin Otolaryngol Allied Sci. 1998;23:27-33.

47. Guzzo M, Locati LD, Prott FJ, Gatta G, McGurk M, Licitra L. Major and minor salivary gland tumors. Crit Rev Oncol Hematol. 2010;74:134-48.

48. Villanueva NL, de Almeida JR, Sikora AG, Miles BA, Genden EM. Transoral robotic surgery for the management of oropharyngeal minor salivary gland tumors. Head Neck. 2013;. doi:10. 1002/hed.23258.

49. Guerissi, JO. "The endoscopic approach to the submandibular gland resection" Operative Atlas of Otorhinolaryngology Head and Neck Surgery: Volume 2. Ed. Bachi T Hathiram, Vicky S Khattar. New Delhi: Jaypee Brothers Medical Publishers. 2013:1362-1365. 